\title{
UMA ANÁLISE DOS CONDICIONANTES DA SATISFAÇÃO, DA DEDICAÇÃO E DO DESEMPENHO DE ESTUDANTES DE CURSOS DE ADMINISTRAÇÃO
}

\author{
AN ANALYSIS OF THE DETERMINANTS OF \\ BUSINESS ADMINISTRATION STUDENTS' SATISFACTION, \\ DEDICATION AND PERFORMANCE
}

Recebido em: 05/09/2013 - Aprovado em: 27/11/2013 Avaliado pelo sistema double blind review Editora Científica: Manolita Correia Lima

\author{
STEPHANIE INGRID SOUZA BARBOZA stephanieisb@gmail.com \\ DIANA LÚCIA TEIXEIRA DE CARVALHO \\ JOÃO BATISTA SOARES NETO \\ FRANCISCO JOSÉ DA COSTA \\ UNIVERSIDADE FEDERAL DA PARAÍBA
}

\begin{abstract}
RESUMO
O objetivo do artigo é analisar os condicionantes da satisfação, da dedicação e do desempenho de estudantes do curso de administração sob uma perspectiva que avalia questões relativas à estrutura do curso e da instituição, além da reputação profissional e do curso. Após uma revisão teórica sobre os tópicos do estudo foi feito um levantamento de campo por meio de um questionário estruturado, aplicado em salas de aula de duas instituições federais de ensino superior de um estado do nordeste brasileiro; a amostra final foi de 176 respondentes. Foram aplicados procedimentos descritivos, de correlação e de análise de regressão, com a finalidade de analisar as influências supostas. Como resultado foi verificado que o desempenho dos estudantes é fundamentalmente definido por seu empenho pessoal nos estudos, praticamente não havendo maiores influências diretas de sua satisfação. Por outro lado, a satisfação dos estudantes, que mostrou ser definida pelos vários fatores analisados, é um determinante consistente do empenho dos estudantes em seus estudos. Palavras chave: educação em Administração; desempenho; dedicação; satisfação.
\end{abstract}

\footnotetext{
ABSTRACT

This paper analyses the determinants of business administration students' satisfaction, dedication and performance by evaluating issues relating to the course and institution structures, in addition to the professional and course reputations. After a theoretical review of these topics, a field survey using a structured questionnaire was carried out at two federal higher education institutions in a northeastern state of Brazil; the final sample contained 176 respondents. Descriptive statistics, correlation, and regression analysis were then employed and the results show that students' performance is fundamentally defined by their personal commitment to studying, and has no direct influence on satisfaction. The students' satisfaction, on the other hand, was proved to be influenced by the various factors analyzed and a consistent determinant of student performance.

Keywords: Administration education; performance; dedication; satisfaction.
} 


\section{INTRODUÇÃO}

O desempenho do aluno universitário normalmente mensurado pelo coeficiente de rendimento escolar das instituições de ensino é uma das grandes metas da formação universitária. Mesmo que o bom desempenho seja oriundo, principalmente, da dedicação do estudante, este é resultado (e indicador) de sucesso da instituição e dos cursos no planejamento e na operacionalização de seus serviços.

A tentativa de ofertar ao estudante um serviço de alta qualidade e não apenas na dimensão do trabalho didático em si, é a preocupação central para a administração das instituições de ensino superior. Entende-se que o sucesso neste intento tem implicações positivas não apenas para os estudantes, mas aos beneficiários externos de uma mão de obra de alto padrão, além de potenciais bons resultados produzidos em termos de pesquisas e inovação (vaduva et al., 20II).

Avaliações exploratórias realizadas pelos autores sinalizam que no Brasil, a oferta de formação superior cresceu a partir da década de 1990 (mais especificamente a partir da lei de Diretrizes e Bases da Educação, de I996); no entanto, a avaliação de práticas sinaliza que este crescimento não foi acompanhado pela adoção de modelos de formação que motivassem melhores desempenhos dos alunos. Esta realidade pode ser notada mediante resultados do Exame Nacional de Desempenho de Estudantes (ENADE), que visa avaliar o rendimento das instituições por meio do desempenho dos seus estudantes, e com base neste observou-se que muitas instituições não alcançaram os melhores conceitos.

Um bom desafio de pesquisa consiste, então, em analisar o que condiciona a dedicação do estudante, o desempenho e a satisfação dos mesmos (HU, 20II). Inicialmente parece razoável acreditar que estas variáveis estão relacionadas, pois uma maior satisfação parece gerar maior dedicação e em conjunto, dedicação e satisfação, que são fatores que promovem melhor desempenho. De forma preliminar, entende-se que a dedicação do estudante tem associação com variáveis de natureza institucional, como também recebe influência adicional da reputação da profissão e do curso. 
Uma boa compreensão destas relações torna-se relevante, na medida em que viabiliza um direcionamento adequado para que gestores de instituições e coordenadores de cursos possam traçar estratégias capazes de melhorar a satisfação, dedicação e desempenho acadêmico dos alunos dos cursos de administração.

Este estudo objetivou analisar fatores de influência sobre a satisfação, a dedicação e o desempenho dos estudantes, com foco específico em cursos de administração. Mais especificamente, o estudo buscou analisar as influências que estes fatores recebem de variáveis relacionadas à estrutura e aos serviços do curso e da instituição, além da reputação profissional e do curso. Entende-se, que, uma vez alcançados estes objetivos, os resultados poderão interessar aos profissionais envolvidos no processo de formação, incluindo docentes e gestores de instituições e cursos.

O conteúdo subsequente apresenta a discussão teórica que embasa este estudo. Posteriormente serão apresentadas as informações acerca dos procedimentos do trabalho de campo. Em seguida, são discorridos os resultados destes e por fim, será feita uma síntese dos resultados, dos quais serão analisadas implicações e limitações. 


\section{REFERENCIAL TEÓRICO}

Conforme resgate histórico de Martins (1989), o contexto da formação do administrador e o início da profissionalização do ensino em Administração no Brasil ganha evidências na década de 1940, objetivando atender a escassez de mão de obra qualificada. Em complemento, atribui-se à regulamentação do ensino pelo governo, e ao crescimento econômico e social, a responsabilidade pelo aumento da demanda nos cursos de administração (NICOLINI, 2003). Questões envolvendo a empregabilidade, entrada no mercado de trabalho, remuneração, recolocação ou readequação são sempre presentes no cenário econômico e gerencial do século xxi, e, consequentemente, na formação de administração (CRUZ; FREITAS JR; SHARLAND, 2009).

É notória, então, a preocupação dos gestores e docentes de cursos de administração em relacionar o processo organizacional produtivo com o processo pedagógico (MOTTA, I983; SARAIVA et al., 20II), ou seja, relacionar as necessidades e demandas organizacionais com estruturas e processos pedagógicos. É, essencialmente, nesta relação que alguns estudos (AKTOUF, 2005; SARAIVA et al., 20II) se concentram, e afirmam que as escolas de administração estimulam um processo educativo pouco crítico e despolitizado, o que mantém o comodismo, o conservadorismo (AKTOUF, 2005) e o rótulo de que a atividade do administrador está relacionada apenas ao executar e não ao pensar (SARAIVA et al., 20II).

Algumas dessas conclusões são atribuídas à estrutura do curso e à ação pedagógica do docente que, em certos casos, não ajustam sua metodologia à heterogeneidade dos discentes (SOUZA-SILVA; DAVEL, 2005). Ainda, é preciso considerar que os cursos de administração devem preparar os alunos para serem capazes de lidar com os desafios oriundos das diversas mudanças enfrentadas pelo cenário contemporâneo, adequando grade curricular e tecnologias de ensino às demandas de mercado (MAмUM; MOHAMAD, 2009). Entretanto, mais do que formar bons administradores, o desafio é promover uma educação que seja relevante para a carreira, mas que também forme pensadores críticos e esclarecidos, motivados a buscar durante toda a vida, desenvolver capacidade analítica e renovar conhecimentos (HAINLINE et al., 20IO). 
Nesse sentido, é importante compreender de que maneira a relação entre estudante e instituição vem se constituindo, principalmente no que diz respeito ao processo de transformação de estudantes em bons profissionais, considerando que o desempenho acadêmico é um fator que, aparentemente, deriva da dedicação e satisfação do aluno, seja com a instituição, com a profissão ou com a própria identificação com o curso. Sendo assim, as instituições de ensino superior mostraram cada vez mais preocupação com a satisfação dos seus alunos, uma vez que, segundo Khan, Ahmed e Nawaz (20II), eles são a ponte entre a instituição e a sociedade, de forma que, quanto mais satisfeito o aluno, melhor seu desempenho e dedicação. São explorados, a seguir, estes aspectos de influência na formação do administrador.

\section{SATISFAÇÃO}

A satisfação do aluno é relacionada às repetidas experiências de vida no campus, uma vez que este ambiente proporciona uma rede de experiências interconectadas que se sobrepõem e influenciam a satisfação dos estudantes como um todo. Desse modo, o que ocorre com os estudantes na sala de aula não é independente das demais experiências que ele vivencia dentro da instituição (ELLIOT; SHIN, 2002).

Petruzzellis, D’Uggento e Romanazzi (2006) colocam que a satisfação do aluno é também dependente das chances de colocação após a graduação e das habilidades adquiridas durante o curso, em uma perspectiva que considera o ensino superior como o início da carreira profissional do indivíduo. Além disso, de acordo com Letcher e Neves (20I0), a satisfação do aluno ajuda a construir autoconfiança, que, por sua vez, proporciona ao mesmo o desenvolvimento de habilidades úteis e a aquisição de conhecimento.

Sendo assim, as instituições de ensino superior em geral, compreendem a satisfação do aluno por meio de dimensões acadêmicas da experiência educacional do estudante, como relação aluno-professor, qualidade do programa e credenciais do corpo docente (ELLIOT, SHIN, 2002). Todavia, é importante considerar que os estudantes sejam capazes de avaliar as dimensões acadêmicas de maneiras distintas. 
Com efeito, as medidas da satisfação do aluno têm sido utilizadas para medir a efetividade dos diferentes serviços (por exemplo, a vida estudantil e a ajuda financeira) e dos programas (como os programas que lidam com demanda de alunos especiais, por exemplo, estudantes internacionais) das universidades, assim como dos próprios programas acadêmicos (LETCHER; NEVES, 20IO). Segundo Gibson (20I0), os atributos relacionados ao programa acadêmico são evidentemente os mais importantes para medir a satisfação geral dos estudantes. Estes incluem a qualidade do ensino e o currículo, bem como a obtenção do aprendizado e dos objetivos de carreira profissional.

Essas medidas podem considerar ainda o contexto da educação (como exemplo, apoio escolar), os recursos do processo (como exemplo, ênfase no conteúdo e feedback), as características estruturais do programa (como exemplo, o tempo do curso) (Ingvarson; meiers; beavis, 2005). De fato, muitos são os aspectos relacionados à experiência acadêmica como um todo, e muitas das pesquisas nesta área têm procurado identificar as características do programa ou dos alunos que impactam na satisfação (LETCHER; NEVES, 2010).

No caso da presente pesquisa, foram utilizadas medidas de avaliação pertinentes ao programa acadêmico de administração, em relação ao curso e sua estrutura (projeto, estrutura, serviço de coordenação). Em termos das medidas de satisfação geral, foi considerada a satisfação do estudante com o curso.

\section{DESEMPENHO}

A motivação e habilidade dos alunos de se responsabilizarem pelo seu próprio aprendizado podem ser consideradas componentes importantes do sucesso acadêmico (DЕмво; ЕAтON, 2000). Alunos que não dependem exclusivamente da relação com o professor como fonte de informação e motivação para aprender, entendem que o seu papel é também se desafiar constantemente, tornando-se estudantes bem sucedidos.

De fato, Dembo e Eaton (2000) colocam que alunos bem sucedidos, mesmo diante de tarefas pouco estimulantes, aparentemente, têm a habilidade de se motivarem para completá-las, ao passo que estudantes 
que mostraram pior desempenho possuem dificuldade em desenvolver habilidades de automotivação. Estas habilidades, em geral, são representadas pelas notas atribuídas às atividades realizadas pelos alunos (forma mais institucionalizada de avaliação do desempenho acadêmico).

Para Kaighobadi e Allen (2008), o desempenho dos alunos é significantemente relacionado a algumas variáveis demográficas básicas, entretanto, os preditores mais fortes para avaliar o sucesso geral dos estudantes são suas notas. Tanto que a pesquisa desenvolvida por Richter (2006) avalia se as notas de um aluno do ensino médio são bons preditores do seu desempenho no ensino superior. Este afirma que as notas são os maiores preditores do desempenho dos estudos acadêmicos dos estudantes, inclusive os de administração.

Cummings, Maddux e Richmond (2008), por sua vez, entendem que as avaliações de desempenho são pertinentes para estimar o desempenho geral dos estudantes de um programa ou instituição. Entretanto, ressaltam a importância de que as atividades de desempenho exponham os estudantes a tarefas que requeiram a construção de conhecimento e não a reprodução de informação, preparando-os para serem pensadores críticos diante das demandas do mercado organizacional.

Sendo assim, os coeficientes de rendimento escolares dos estudantes podem ser considerados como medida suficientemente adequada de desempenho acadêmico. Sulaiman e Mohezar (2006) sugerem que há uma relação entre a experiência de trabalho, a disciplina da graduação, o rendimento médio escolar e variáveis demográficas como idade e gênero com o desempenho do graduando.

No caso desta pesquisa, o desempenho médio do aluno e a autoavaliação (postura, seriedade e compromisso) foram as variáveis utilizadas para medir a avaliação do estudante. São ainda consideradas como variáveis de influência do desempenho acadêmico questões relativas à estrutura do curso e da instituição, além da reputação profissional e do curso, considerando que, segundo Gibson (20I0), as experiências dos alunos com professores, aulas, práticas administrativas, facilidades físicas do campus e ambiente social são fatores que influenciam a avaliação geral da experiência acadêmica dos estudantes. 


\section{DEDICAÇÃO}

Alguns autores (AXELSON; FLICK, 2OII; DAVIDSON; AMENKHIENAN, 2OII) concordam que não há uma definição simples para o termo "engajamento do estudante", o qual, nesta pesquisa, foi chamado de dedicação do aluno. Todavia, entende-se que dedicação refere-se a quão o aluno está envolvido ou interessado com o seu aprendizado ou a quão conectado ele se sente com suas aulas, sua instituição e uns com os outros (AXELSON; FLICK, 20II).

Arend (2004) define dedicação como sendo simplesmente o tempo e o esforço gasto em atividades, o que se tornou uma maneira estabelecida de compreender a experiência e a qualidade de aprendizado do estudante no ensino superior. A dedicação dos estudantes, portanto, é algo que pode melhorar tanto seu aprendizado quanto seu desempenho (DAvidson; AMENKhiENAN, 20II). Afinal, aparentemente, quanto mais o aluno estuda um tema, mais ele está propenso a aprendê-lo (CARINI; KUH; KLEIN, 2006).

Neste sentido, a dedicação do aluno acerca de atividades educacionais, dentro ou fora da sala de aula, pode contribuir para o seu aprendizado e seu desenvolvimento pessoal, estando diretamente ligada ao sucesso acadêmico (HU, 20II). Entretanto, Axelson e Flick (20II) ressaltam ser importante considerar que níveis, tipos ou resultados de dedicação diferem entre grupos de estudantes e que a dedicação pode ser simplesmente o subproduto de um ambiente de aprendizagem no qual o aluno se sinta adaptado.

Caso a dedicação esteja vinculada ao ambiente de aprendizado e não apenas às variáveis dependentes da dedicação, como aprendizado e seus resultados (notas e coeficiente de rendimento escolar), um conjunto de fatores relacionados às características de estrutura do campus e das experiências acadêmicas estaria interferindo para que o aluno se sentisse motivado a se dedicar academicamente (AXELSON; FLICK, 20II). Deste modo, é pertinente que as medidas utilizadas nesta pesquisa para avaliar a dedicação pessoal dos estudantes ao curso de administração estejam relacionadas à estrutura do curso e da instituição, bem como da reputação profissional e do curso. 


\section{MÉTODO}

Esta seção apresenta as decisões do trabalho de campo, com foco específico na mensuração, no design, e nos procedimentos de análise de dados. Para a medição, a opção preferencial foi por medidas de um só item para os construtos de base, a saber: desempenho, satisfação, autoavaliação, dedicação, avaliação do curso (projeto, estrutura, serviço de coordenação) e de professores (didática, compromisso e relacionamento).

Especificamente para os construtos reputação da profissão e identificação com a profissão, foram utilizados múltiplos itens (por mensuração refletiva), segundo a escala utilizada em Costa (2008), que foi escolhida tendo em vista, principalmente, a validade de conteúdo e a indicação prévia de validade estatística. Os itens estão no Anexo I e na seção de resultados são apresentadas as indicações relativas à análise da estrutura psicométrica, considerando as indicações de Hair et al. (2009) e Costa (20II) sobre as medidas de referência da análise fatorial e de consistência interna (кмо desejável maior que o,6; estatística de Barttlet desejável significativa a p<0,05; escores fatoriais desejáveis acima de 0,5; e alpha de Cronbach desejável acima de 0,6$)$.

O desempenho dos estudantes foi medido pelo coeficiente de rendimento acadêmico informado pelos alunos (que é uma média global das notas destes computados em parte das instituições de ensino superior brasileiras, e principalmente nas universidades federais).

O instrumento de coleta de dados apresentou os itens dos construtos por meio de afirmações, com aferição das medidas pelo nível de concordância, no modelo de escala de Likert de ir pontos (de o a io). Além de apresentar tais escalas, o instrumento de coleta de dados apresentou questões referentes ao perfil socioeconômico e demográfico dos sujeitos respondentes.

Os dados foram coletados nas salas de aula de duas instituições federais de ensino superior de um estado do nordeste brasileiro, entre os estudantes do curso de graduação em Administração que estavam cursando a partir do segundo semestre (uma vez que estes sujeitos têm acesso ao seu coeficiente de rendimento escolar, além de já se relacionarem com as 
estruturas da instituição de ensino). A partir deste procedimento, foram obtidos I80 questionários que, em sequência, foram tabulados no SPSS. Em procedimentos preliminares de análise, foram excluídos os questionários que apresentavam alguma restrição para operacionalização estatística, restando 176 instrumentos adequados para esta.

Os procedimentos de análise iniciaram-se com a descrição das variáveis por meio das medidas de posição (média e mediana), das medidas de dispersão (desvio padrão), e com a verificação dos valores de assimetria e curtose. Em seguida, foram aplicados procedimentos paramétricos e não paramétricos de análise de influência, com base na correlação de Pearson e Spearman, respectivamente. Por fim, foi procedida uma análise de regressão, segundo o modelo normal linear múltiplo, no intuito de verificar os condicionantes dos construtos dedicação, satisfação e desempenho dos sujeitos respondentes. Todos os procedimentos definidos ocorreram com base na literatura especializada (CONOVER, I999; HAIR et al., 2009; LATTIN; CARROL; GREEN, 2OII), e serão feitos detalhamentos e especificações na medida em que forem apresentados. 


\section{RESULTADOS}

Esta seção apresenta os resultados dos procedimentos estatísticos, em três blocos: primeiramente, será exposta a descrição das variáveis (média, mediana, desvio padrão, assimetria e curtose); em seguida, serão feitas as análises de influência (análise de correlação paramétrica e não paramétrica), ao final, serão elaborados outros comentários relevantes.

\section{MEDIDAS DESCRITIVAS}

A amostra final pode ser caracterizada como equilibrada quanto ao gênero ( $48,3 \%$ masculino e $51,7 \%$ feminino), sendo que $83 \%$ dos respondentes apresentaram 'solteiro' como estado civil; a renda média familiar predominante foi até $\mathrm{R} \$ 3.000,00$ (68\%), e a idade predominante foi entre 2I e 23 anos (40\%). Conforme estes dados pode-se afirmar que a amostra apresenta um desejável grau de heterogeneidade, o que indica sua adequação para a realização das análises subsequentes.

Nestes termos, inicialmente, foram extraídas as medidas descritivas de posição (média e mediana), dispersão (desvio padrão) e forma (assimetria e curtose) das variáveis associadas ao estudante e à instituição. Os resultados estão na Tabela I, divididos em três blocos: avaliação do próprio aluno, avaliação da instituição e do curso, e satisfação e dedicação ao curso.

Tabela I Avaliações associadas ao curso

\begin{tabular}{|llllll|}
\hline \begin{tabular}{l} 
Variáveis \\
\hline Variáveis de avaliação do estudante
\end{tabular} & Média & Mediana & Desvio & Assim. & Curtose \\
\hline $\begin{array}{l}\text { Desempenho médio do aluno } \\
\text { Autoavaliação (postura, seriedade e }\end{array}$ & 7,45 & 7,50 &, 91 &,- 65 &, 59 \\
\hline $\begin{array}{l}\text { compromisso) } \\
\text { Variáveis de avaliação do curso e sua estrutura }\end{array}$ & 7,46 & 8,00 & 1,44 &,- 55 &,- 07 \\
\hline $\begin{array}{l}\text { Prestígio de mercado do curso (da instituição) } \\
\text { Atuação da coordenação }\end{array}$ & 7,93 & 8,00 & 1,64 &,- 68 &,- 03 \\
\hline
\end{tabular}




\begin{tabular}{|c|c|c|c|c|c|}
\hline Variáveis & Média & Mediana & Desvio & Assim. & Curtose \\
\hline $\begin{array}{l}\text { Qualidade geral do projeto pedagógico do } \\
\text { curso }\end{array}$ & 6,25 & 7,00 & 1,86 &,- 72 & ,22 \\
\hline Estrutura física do curso & 4,22 & 4,00 & 2,19 &,- 05 &,- 60 \\
\hline Didática dos professores do curso & 6,39 & 7,00 & 1,75 &,- 42 &,- 13 \\
\hline Compromisso dos professores do curso & 6,49 & 7,00 & 1,89 &,- 56 &,- 02 \\
\hline $\begin{array}{l}\text { Relacionamento dos professores com os } \\
\text { alunos }\end{array}$ & 6,71 & 7,00 & 1,82 &,- 84 & 1,15 \\
\hline \multicolumn{6}{|l|}{ Satisfação e dedicação com o curso } \\
\hline Satisfação geral com curso & 6,88 & 7,00 & 1,92 &,- 75 &, 60 \\
\hline Nível de dedicação pessoal ao curso & 7,44 & 8,00 & 1,49 &,- 45 &,- 49 \\
\hline
\end{tabular}

No primeiro bloco foi apresentada a avaliação dos estudantes em termos de sua nota (pelo coeficiente de rendimento acadêmico) e da autoavaliação (em termos de postura, seriedade e compromisso), aqui se observou que as avaliações dos alunos estão em nível moderado em termos de média e mediana sendo baixa a dispersão, o que sinaliza que há boa convergência nas respostas dos sujeitos. As medidas de posição sinalizam distribuições de origem das amostras como sendo simétricas e mesocúrticas (é aquela que apresenta um nível médio de curvatura), dentro de padrões de normalidade.

No segundo bloco, foram expostas as avaliações quanto ao curso e à instituição. Aqui, as médias e medianas foram mais baixas, sendo verificado ainda um valor destacadamente baixo na avaliação da estrutura física da universidade (a exceção ficou por conta do nível de prestígio externo atribuído ao curso). As medidas de dispersão foram já um pouco mais elevadas, mas ainda estão em nível moderado, havendo sinalização de maior dispersão de opiniões (comparativamente ao primeiro bloco de avaliação). As medidas de formato seguem o padrão da primeira avaliação. Um destaque aqui está nas três variáveis de avaliação dos professores, que foram todas muito próximas (inclusive com medianas idênticas em todas) e níveis de dispersão também próximos. 
Em geral, o nível de satisfação do estudante com o curso foi moderado, com valores de média a mediana em torno de 7,00, com desvio padrão moderado. O nível de dedicação declarado foi já mais elevado com desvio padrão também moderado. Nos dois casos, as medidas de formato seguem o padrão observado nas variáveis das demais dimensões.

No caso dos construtos reputação percebida na profissão e identificação pessoal com a profissão, as medições foram procedidas por múltiplos itens, supostos terem relação refletida em relação ao construto de referência. Para análise de sua adequação, foram procedidas duas iterações de análise fatorial, uma com cada bloco de itens.

As medidas descritivas e os escores fatoriais estão indicados no anexo deste artigo, e pelo que se observou a boa estrutura fatorial está assegurada. De fato, de acordo com a literatura especializada (HAIR et al., 2009; LATTIN; CARROL; GREEN, 2OII) os testes indicaram boa adequação da amostra de variáveis para análise fatorial (к мо >0,7 e significância do qui-quadrado de Batlett menor que 0,05), assim como a variância extraída (desejável ser maior que 0,5 ) mostrou que, em cada caso, o construto subjacente explicava mais de $50 \%$ da variação do total de itens (estes resultados estão nas notas da Tabela 2).

Os escores fatoriais foram todos acima do limite requerido de adequação (desejáveis serem maiores que o,5), sinalizando que todos eles possuíam correlação no mínimo moderada com o fator (as medidas estão em anexo). Convém ainda ressaltar a coerência deste resultado com aquele observado no estudo de origem da escala (COSTA, 2008), que atestou boa estrutura psicométrica aferida pelos valores de alpha de Cronbach, que foram de o,88 para prestígio (aqui de o,86), e de o,89 para identificação (aqui o,93). Estes resultados asseguram condições para agregação das variáveis em uma só medida para representar os construtos. Este procedimento foi realizado pela extração das médias dos escores para cada conjunto de variáveis por respondente. Os resultados descritivos estão na Tabela 2. 
$\&$ OUTROS

Tabela 2 Medidas de reputação e identificação com a profissão

\begin{tabular}{|lllllll|}
\hline Variáveis & Média & Mediana & Desvio & Assim. & Curtose \\
\hline Reputação percebida na profissão* & 6,97 & 7,43 & 1,82 &,- 79 &, 30 \\
\hline Identificação pessoal com a profissão** & 7,78 & 8,20 & 1,81 & $-1,08$ &, 91
\end{tabular}

* KMO: 0,919; Teste de Bartlett: $\chi^{2}=948,49, \mathrm{p}<0,001$; Variância extraída: 70,84\%; Menor escore: 0,711

${ }^{*}$ KMO: 0,863; Teste de Bartlett: $\chi^{2}=814,74, p<0,001$; Variância extraída: 81,03\%; Menor escore: 0,866

As medidas descritivas de reputação apontam, em geral, valores moderados para média e mediana, havendo aqui valores de dispersão mais elevados, o que sinaliza que os respondentes possuem maior variação de posições. Em relação às variáveis de identificação pessoal com a profissão, as medidas de posição são um pouco mais elevadas, e possuem, igualmente, nível moderado de dispersão. Em relação às medidas de assimetria e curtose, é possível observar variações de resultados, surgindo mais sinalização de assimetria à esquerda e na variável agregada de identificação (nas demais medidas há sinalização de que as variáveis são oriundas de uma população com distribuição normal nestes tópicos).

\section{ANÁLISE DE INFLUÊNCIA}

Após a análise descritiva, foi realizada uma primeira verificação da associação bivariada entre as variáveis centrais do estudo, por meio da extração das medidas de correlação de Pearson e Spearman (esta última é adequada para medições com escalas intervalares, como as que são utilizadas aqui, e seu uso capta variações conjuntas inclusive de natureza não linear). Os resultados estão indicados na Tabela 3. 
Tabela 3 Medidas de correlação

\begin{tabular}{|c|c|c|c|c|c|c|}
\hline \multirow[t]{2}{*}{ Variáveis } & \multicolumn{2}{|c|}{$\begin{array}{l}\text { Desempenho } \\
\text { Pessoal }\end{array}$} & \multicolumn{2}{|c|}{$\begin{array}{l}\text { Satisfação com o } \\
\text { curso }\end{array}$} & \multicolumn{2}{|c|}{$\begin{array}{l}\text { Dedicação ao } \\
\text { curso }\end{array}$} \\
\hline & 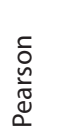 & 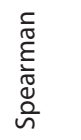 & 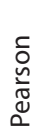 & 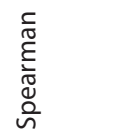 & 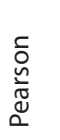 & 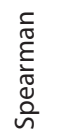 \\
\hline Satisfação com o curso & 17 &, $12^{*}$ & & & & \\
\hline Dedicação ao curso & ,45 & ,42 &, 51 &, 54 & & \\
\hline Reputação da profissão &, $13^{*}$ &, $08^{*}$ & 64 & ,61 & ,42 & ,41 \\
\hline Identificação pessoal & $14^{*}$ & ,15 & 42 & ,43 & 42 & ,42 \\
\hline Prestígio do curso &, $12^{*}$ &, $12^{*}$ & 60 & ,62 & ,42 & ,43 \\
\hline Autoavaliação & ,48 & ,43 & ,34 & ,33 & ,73 & ,71 \\
\hline Atuação da coordenação & 17 &, $12^{*}$ &, 42 &, 43 & ,26 & ,27 \\
\hline Qual. do projeto pedagógico &, $00^{*}$ &,$- 01^{*}$ & ,67 & ,65 & 31 & ,37 \\
\hline Estrutura do curso &,$- 01^{*}$ &,$- 02 *$ &, 46 &, 43 & 16 & ,16 \\
\hline Didática dos professores &, $02^{*}$ &, $01^{*}$ &, 55 &, 55 & ,28 & ,28 \\
\hline Compromisso dos professores &, $15^{*}$ &, $14^{*}$ & ,56 &, 56 &, 37 & ,38 \\
\hline Relacionamento dos professores &, $08^{*}$ &, $02^{*}$ &, 53 &, 52 & 29 & ,29 \\
\hline
\end{tabular}

* Valores estatisticamente nulos, a $\mathrm{p}>0,05$.

Conforme se observa, e levando em conta a expectativa de que alunos mais satisfeitos se dedicam mais ao curso e possuem melhor desempenho, os resultados mostraram as seguintes implicações:

- Em relação aos fatores que influenciam o desempenho do aluno (em termos da nota média), as medidas de correlação mostraram que é a dedicação pessoal ao curso e a autoavaliação que explicam o desempenho, o que se confirma nas duas verificações de correlação (veja que as variáveis associadas à coordenação e satisfação mostraram correlação baixa ou até nula (por Spearman), ou seja, se estas variáveis influenciam o desempenho, tal influência é modesta e marginal). Isto sinaliza claramente que o que de fato impacta no desempenho do aluno é sua dedicação e responsabilidade, o que, em boa medida, reafirma algo que a experiência corrente mostra. Ademais, os elementos estruturantes do curso mostraram possuir 
impacto apenas marginal. Isto não indica que estes aspectos não são relevantes, pois podem influenciar a dedicação do estudante ao curso ou a sua satisfação.

- Em relação à satisfação com o curso, observou-se que esta teve correlação positiva com todas as variáveis, de maneira destacada com as variáveis associadas à reputação percebida na profissão, da reputação externa do curso e da estrutura curricular (que tiveram correlações maiores que o,6), e é bem destacada nas correlações com as três variáveis relativas aos professores (com correlações acima de 0,5 ). Em princípio, é possível concluir que todas estas variáveis possuem impacto moderado na construção da satisfação do estudante com seu curso. Isto reafirma o entendimento corrente de que um aluno fica satisfeito com seu curso se tiver (I) uma clara percepção de boa reputação externa deste e da profissão, (2) um bom projeto pedagógico com uma matriz curricular de elevado impacto em termos de aprendizado, e (3) uma boa qualidade docente (em termos de didática, compromisso e relacionamento).

- Por fim, em relação à dedicação do estudante ao seu curso, observou-se que todas as correlações foram positivas, destacando-se aí a autoavaliação com correlação acima de o,7, em um nível mais elevado. A sinalização é de que a dedicação é, na verdade, mais fortemente condicionada pelo nível de responsabilidade pessoal que o aluno já possui. Um aspecto de destaque é a influência de professores que nas três dimensões avaliadas, teve correlações já baixas (em torno de o,3), ou seja, em geral, professores pouco influenciam a dedicação do aluno (este resultado é controverso, pois é esperado que bons professores promovam maior motivação nos alunos; a negação deste resultado, preliminarmente, sugere a necessidade de maiores explorações desta relação).

Para explorar adicionalmente os resultados foram procedidas extrações de regressões (pelo modelo normal linear múltiplo), na tentativa de analisar melhor o que pode ser condicionante de desempenho e satisfação dos estudantes. Em cada iteração foi adotado o procedimento backward, que testa o modelo global e segue excluindo as variáveis com influência 
sobre a variável resposta estatisticamente nula. Assim, todas as variáveis associadas ao curso, à reputação com a profissão, à identificação pessoal com a profissão, à autoavaliação e à satisfação geral com o curso foram inseridas para previsão da dedicação ao curso; na sequência, a variável satisfação foi posta como a variável resposta, e as demais como variáveis preditoras; por fim, todas as variáveis foram colocadas como preditoras do desempenho dos estudantes.

Os modelos finais foram avaliados em seus pressupostos relativos aos erros (estimados pelos resíduos de regressão), e se mostraram adequados. No entanto, optou-se por não exibir aqui os resultados, seguindo a tradição de pesquisas semelhantes a esta aqui desenvolvida. Os resultados das medidas de influência estão consolidados na Tabela 4.

Tabela 4 Resultados da regressão (método backward)

\begin{tabular}{|c|c|c|c|c|c|c|c|c|c|}
\hline \multirow[t]{2}{*}{$\begin{array}{l}\text { Variáveis } \\
\text { preditoras }\end{array}$} & \multicolumn{3}{|c|}{$\begin{array}{l}\text { dedicação ao curso } \\
\left(r^{2}=0,613\right)\end{array}$} & \multicolumn{3}{|c|}{$\begin{array}{l}\text { satisfação geral com } \\
\text { curso }\left(r^{2}=0,680\right)\end{array}$} & \multicolumn{3}{|c|}{$\begin{array}{l}\text { desempenho do aluno } \\
\left(r^{2}=0,249\right)\end{array}$} \\
\hline & $\boldsymbol{\beta}$ & $\mathbf{t}$ & p-valor & $\beta$ & $\mathbf{t}$ & p-valor & $\beta$ & $\mathbf{t}$ & p-valor \\
\hline $\begin{array}{l}\text { Satisfação geral } \\
\text { com curso }\end{array}$ & ,225 & 3,491 & ,001 & & & & & & \\
\hline Autoavaliação & ,621 & 12,121 &, 000 & ,094 & 1,979 & ,050 & ,318 & 3,267 & ,001 \\
\hline $\begin{array}{l}\text { Reputação da } \\
\text { profissão }\end{array}$ & 111 & 1,757 & ,081 & ,351 & 6,129 &, 000 & & & \\
\hline $\begin{array}{l}\text { Prestígio do } \\
\text { curso }\end{array}$ & & & & 283 & 5,306 &, 000 & & & \\
\hline $\begin{array}{l}\text { Estrutura do } \\
\text { curso }\end{array}$ & & & & 120 & 2,341 & ,020 & & & \\
\hline $\begin{array}{l}\text { Qual. do projeto } \\
\text { pedagógico }\end{array}$ & & & & ,307 & 4,948 &, 000 & & & \\
\hline $\begin{array}{l}\text { Compromisso } \\
\text { dos professores }\end{array}$ & & & & ,129 & 2,262 & ,025 & & & \\
\hline $\begin{array}{l}\text { Dedicação ao } \\
\text { curso }\end{array}$ & & & & & & & 218 & 2,235 & ,027 \\
\hline
\end{tabular}

Pelo que se observou na previsão da dedicação pessoal ao curso, o modelo final mostrou influência significativa de duas variáveis apenas (satisfação com o curso e autoavaliação), com 6I,3\% de sua variação explicada pelas variáveis independentes, que já pode ser considerado elevado $\left(\mathrm{R}^{2}=0,6 \mathrm{I} 3\right)$. Em 
um nível marginal é atestada a influência da identificação do aluno com a profissão.

Em relação à satisfação do aluno, ao final, foi obtida influência consolidada de seis fatores (autoavaliação, reputação da profissão, qualidade do projeto pedagógico, estrutura do curso, prestígio externo do curso e compromisso dos professores); em nível global, o construto teve $68,0 \%$ de sua variação explicada pelas variáveis antecedentes, ou seja, a satisfação tem sua variação fortemente explicada por estes seis fatores.

Por fim, somente dois aspectos influenciaram o desempenho dos estudantes (autoavaliação e dedicação pessoal ao curso), que tem somente $24,9 \%$ de sua variação total explicada por estes fatores. Isto sugere, inicialmente, a existência de vários outros fatores que impactam no desempenho do estudante como: sua formação anterior, seu nível pessoal de inteligência, além de outros que não entraram na avaliação do estudo. Ainda assim, destacou-se o fato de algumas variáveis não terem assegurado influência. 


\section{COMENTÁRIOS ADICIONAIS}

O conteúdo apresentado nos resultados chama a atenção em alguns aspectos. Primeiramente, no resultado descritivo é notável a baixa avaliação do aluno quanto ao curso e à instituição, e também quanto à estrutura física do curso. Estes aspectos são geradores de motivação do aluno, o que se reafirma tanto nos dados quanto nas análises de Carraher, Carraher e Schliemann (1989) e Martini e Del Prette (2005). Verificou-se também uma baixa média atribuída à qualidade geral do projeto pedagógico do curso, o que, segundo os dados é um elemento de associação moderada com a satisfação dos estudantes. Este é certamente um problema a ser refletido tanto pela instituição de base da pesquisa, quanto para outras que podem vivenciar problemas semelhantes levando em conta que não deve haver descompasso entre conteúdo programático e o método de ensino adotado, o que pode gerar uma frustração do aluno quanto à inadequada preparação recebida pela IES para o mercado de trabalho (MELO; BORGES, 2005).

Do ponto de vista da análise de influência, no que tange ao desempenho discente os dados apontaram uma influência somente de dedicação pessoal ao curso e de autoavaliação, o que pode se explicar pelo estudo de Weiner (1985). Para este autor três dimensões (internalidade, estabilidade e controlabilidade) explicam as causas do sucesso ou fracasso escolar, e ele acrescenta ainda que a capacidade e o esforço são causas internas ao sujeito e, como tal, influenciadoras das reações afetivas, de autoestima e de autoconceito, o que tem impacto no rendimento. Também, há uma reafirmação da visão de Martini e Del Prette (2005), que pontuam que o aspecto comportamental tem papel determinante na motivação e no rendimento do aluno, o que pode justificar a relação da autoavaliação (postura, seriedade e compromisso) com o desempenho do aluno.

Com relação à satisfação com o curso, verificou-se maior influência de três variáveis. Duas delas, prestígio do curso e reputação da profissão, estão associadas à avaliação mais externa (COSTA, 2008); a outra foi o projeto pedagógico do curso, e destaca o aspecto ligado ao conteúdo educacional da análise. Por estas três variáveis, é entendido que a satisfação do discente 
em aprender acontece graças às expectativas externas e à relevância do conteúdo estudado pelo mesmo, além de um projeto consistente e bem direcionado.

Por fim, na esfera da dedicação do estudante ao curso (e por consequência no seu desempenho), a variável autoavaliação foi a mais influenciadora. Esta semelhança pode ser fundamentada pela internalidade proposta por Weiner (1985) e pelo aspecto comportamental de Martini e Del Prette (2005). Em ambos os casos, há o entendimento de que a capacidade e o esforço de cada um são internos e não de fora para dentro, o que bem embasa o conceito de desempenho e dedicação.

Em geral, observou-se que as associações esperadas entre as diversas variáveis estudadas e o desempenho (ou pelo menos correntemente enunciadas como formadoras do bom desempenho discente) têm efeito indireto. De fato, dedicação e esforço pessoal são os definidores diretos do desempenho; mas estas variáveis são, por sua vez, influenciadas por fatores direcionadores de satisfação tais como: o projeto do curso, sua estrutura e seus docentes.

Embora o estudo não seja conclusivo, por seu design e por suas limitações, é destacada a ausência de características docentes na definição (pela regressão) das variáveis de saída analisadas (satisfação, dedicação e desempenho). A análise bivariada assegura a influência da didática, relacionamento e compromisso docente com satisfação e dedicação (e não com desempenho), porém na análise conjunta esta influência se dispersa com a influência das demais variáveis. Apesar disto, acredita-se que as variáveis docentes estão entre as principais formadoras de bom desempenho; provavelmente, o procedimento metodológico aqui adotado não viabilizou esta visualização, sendo recomendada a adoção de outros métodos para uma verificação mais segura deste resultado. 


\section{CONSIDERAÇÕES FINAIS}

O estudo teve por finalidade analisar aspectos da oferta e da operacionalização do serviço de formação de estudantes universitários de administração, especificamente variáveis relacionadas à estrutura, aos professores, e condicionantes associados à profissão. Tudo isto foi analisado em seu impacto sobre a satisfação, a dedicação e o desempenho dos estudantes de cursos de administração.

Foi possível observar que o desempenho dos estudantes é fundamentalmente definido por seu empenho pessoal nos estudos, praticamente não havendo maiores influências diretas da satisfação. Por outro lado, foi identificado que a satisfação dos estudantes é um determinante consistente do empenho dos mesmos em seus estudos.

Também foi possível mostrar que a satisfação dos discentes é definida pelos vários fatores analisados: estrutura física, desenvolvimento das atividades, reputação do curso e da profissão. Com isto, conclui-se que, em conjunto, as diversas variáveis analisadas são influenciadoras do desempenho dos alunos, embora sejam mediadas pela satisfação dos estudantes e pelo nível de dedicação dos mesmos no curso.

Estes resultados estão coerentes com o que já se conhece na literatura especializada, porém têm a fragilidade de serem uma suposição não testada de forma mais ampla. Com efeito, o teste de relações simultâneas requereria outros procedimentos estatísticos, como modelagem de equações estruturais ou de equações simultâneas. Isto não foi possível, uma vez que a estratégia de mensuração adotada não previa este tipo de operacionalização de dados. Neste sentido, fica recomendado para outros estudos a verificação destas relações por estes procedimentos ou outros que se mostrem adequados para efeito de análise.

Outra limitação do estudo concerne à amostra e ao procedimento de amostragem. De fato, a amostra, embora tenha viabilizado o teste das relações de forma consistente, ainda pode ser considerada pequena. Ademais, o contexto de local sugere que os resultados podem não se repetir em outros locais de aplicação da coleta de dados. Embora o estudo tenha 
\& OUTROS

alcançado seus objetivos, por estas restrições, acredita-se que as relações testadas poderão ter sua compreensão melhorada (em especial a relação de influência simultânea) com outros estudos que possuem amostras maiores e um número mais amplo de instituições.

Acredita-se, no entanto, que os resultados têm sentido do ponto de vista prático, em especial se for considerada a experiência corrente do processo de formação superior em Administração no Brasil. Por esta razão, o estudo poderá ser útil a coordenadores de cursos e a gestores de instituições de educação superior, na medida em que ficou enfatizado a relevância da satisfação dos estudantes no processo de formação, assim como a necessidade de se buscar continuamente desenvolver nos alunos um senso de dedicação e maior empenho. 


\section{REFERÊNCIAS}

AKTOUF, O. Ensino de administração: por uma pedagogia para a mudança. Organizações \& Sociedade, Salvador, v. 12, n. 35, p. 151-159, 2005.

AREND, B. D. Bottom Line - New Patterns of Student Engagement. About Campus, v. 9, n. 1, p. 30-32, 2004.

AXELSON, R. D.; FLICK, A. Defining Student Engagement. Change: The Magazine of Higher Learning, v. 43, n. 1, p 38-43, 2011.

CARINI, R. M.; KUH, G. D.; KLEIN, S. P. Student Engagement and Student Learning: Testing the Linkages. Research in Higher Education, v. 47, n. 1, p. 1-32, 2006.

CARRAHER, T. N.; CARRAHER, D. W.; SCHLIEMANN, A. D. Na vida dez, na escola zero. São Paulo, Brasil: Cortez, 1989.

CONOVER, W. J. Practical Non Parametric Statistics. 3.ed. New York: John Wiley \& Sons, 1999.

COSTA, F. J. Formação em Administração na Perspectiva do Aluno: Valor Percebido no Curso, Percepção do Prestígio e Identificação com a Profissão. Revista Ciências Administrativas, v. 14, n. 1, p. 151-163, 2008.

COSTA, F. J. Mensuração e desenvolvimento de escalas: aplicações em Administração. Rio de Janeiro: Ciência Moderna, 2011.

CUMMINGS, R; MADDUX, C. D.; RICHMOND, A. Curriculum-embedded Performance Assessment in Higher Education: Maximum Efficiency and Minimum Disruption. Assessment \& Evaluation in Higher Education, v. 33, n. 6, p. 599-605, 2008.

CRUZ, B; FREITAS JR, A; SHARLAND, E. Reputação como Fonte de Vantagem Competitiva: Uma Análise Empírica a Partir de uma Instituição de Ensino Superior. In: EnEPQ, 2., 2009. Curitiba. Anais... Curitiba: Anpad, 2009.

DAVIDSON, D; AMENKHIENAN, F. Student Engagement Should Be Fundamental. Franklin Business \& Law Journal, v. 2, n.1, p 39-50, 2011.

DEMBO, M. H.; EATON, M. J. Self-Regulation of Academic Learning in Middle-Level Schools. The Elementary School Journal, v. 100, n. 5, p. 473-490, 2000.

ELLIOT, K. M.; SHIN, D. Student Satisfaction: An Alternative Approach to Assessing this Important Concept. Journal of Higher Education Policy and Management, v. 24, n. 2, p. 197-209, 2002.

GIBSON, A. Measuring Business Student Satisfaction: A Review and Summary of the Major Predictors. Journal of Higher Education Policy and Management, v. 32, n. 3, 251-259, 2010.

HAINLINE, L.; GAINES, M.; FEATHER, C. L.; PADILLA, E.; TERRY, E. Changing Students, Faculty, and Institutions in the Twenty-First Century. Peer Review, v. 12, n. 3, p. 7-10, 2010. 
HAIR Jr., J.; BLACK, W. C.; BABIN, B. J.; ANDERSON, R. E.; TATHAM, R.L. Análise Multivariada de Dados. 6.ed. Porto Alegre: Bookman, 2009.

HU, J. Reconsidering the Relationship between Student Engagement and Persistence in College. Innovative Higher Education, v. 36, n. 2, p. 97-106, 2011.

INGVARSON, L.; MEIERS, M.; BEAVIS, A. Factors affecting the impact of professional development programs on teachers' knowledge, practice, student outcomes \& efficacy. Education Policy Analysis Archives, v. 13, n. 10, p. 1-29, 2005

KAIGHOBADI, M.; ALLEN, M. T. Investigating Academic Success Factors for Undergraduate Business Students. Decision Sciences Journal of Innovative Education, v. 6, n. 2, p. 427-436, 2008.

KHAN, M. M.; AHMED, I.; NAWAZ, M. M. Student's Perspective of Service Quality in Higher Learning Institutions; Anevidence Based Approach. International Journal of Business and Social Science, v. 2, n. 11, p. 159-164, 2011.

LATTIN, J.; CARROL, J. D.; GREEN, P. E. Análise de Dados Multivariados. São Paulo: Cengage Learning, 2011.

LETCHER, D. W.; NEVES, J. S. Determinants of Undergraduate Business Student Satisfaction. Research in Higher Education Journal, p 1-26. 2010.

MAMUM, M. A.; MOHAMAD, A. B. Management Education for Contemporary Challenges: The Role of Business School. European Journal of Scientific Research, v.30, n.4, p. 649-661, 2009.

MARTINI, M. L.; DEL PRETTE, Z. A. P. Atribuições de Causalidade e Afetividade de Alunos de Alto e Baixo Desempenho Acadêmico em Situações de Sucesso e de Fracasso Escolar. Revista Interamericana de Psicologia, v. 39, n. 3, p. 355-368, 2005.

MARTINS, C. B. Surgimento e expansão dos cursos de administração no Brasil (19521983). Ciência e Cultura, v. 41, n. 7, p. 663-676, 1989.

MELO, S. L.; BORGES, L. O. Transição Universidade-Mercado de Trabalho na Ótica do Jovem. In: EnANPAD, 29., 2005. Brasília. Anais... Brasília: Anpad, 2005.

MOTTA, F. C. A. P. A questão da Formação do Administrador. Revista de Administração de Empresas, v. 23, n. 4, p. 72-85, 1983.

NICOLINI, A. Qual Será o Futuro das Fábricas de Administradores? Revista de Administração de Empresas, v. 43, n. 2, p. 44-54, 2003.

PETRUZZELLIS, L.; D’UGGENTO, A. M.; ROMANAZZI, S. Student Satisfaction and Quality of Service in Italian Universities. Managing Service Quality, v. 16, n. 4, p. 349-364, 2006.

RICHTER, A. Intertemporal Consistency of Predictors of Student Performance: Evidence from a Business Administration Program. Journal of Education for Business, v. 82, n. 2, p. 88-93, 2006.

SARAIVA, L. A. S.; SOUZA, C. S.; FERRERO, A. M.; COIMBRA, K. E. R. A Moral 
do Super Homem e a Formação do Administrador. In: EnEPQ, 3., 2011. João Pessoa. Anais... João Pessoa: Anpad, 2009.

SOUZA-SILVA, J. C.; DAVEL, E. Concepções, Práticas e Desafios na Formação do professor: Examinando o Caso do Ensino Superior de Administração no Brasil. Organizações \& Sociedade, v. 12, n. 35, p. 113-134, 2005.

SULAIMAN, A.; MOHEZAR, S. Student Success Factors: Identifying Key Predictors. Journal of Education for Business, v. 81, n. 6, p 328-333, 2006.

VADUVA, S. A.; FOTEA, W.; CORCEA, M.; LAZAR, M. Beyond Satisfaction: Engaging Students and Faculty in a Romanian Business School. Marketing Management Journal, v. 21, n.1, p. 207-213, 2011.

WEINER, B. An Atribucional Theory of Achievement, Motivation, and Emotion. Psychological Review, v. 92, n. 4, p. 548-573, 1985. 


\section{ANEXO I MEDIDAS DE REPUTAÇÃO E IDENTIFICAÇÃO COM A PROFISSÃO}

\begin{tabular}{|c|c|c|c|c|c|c|}
\hline variáveis & escore & média & mediana & desvio & assim. & curtose \\
\hline \multicolumn{7}{|c|}{$\begin{array}{l}\text { Reputação percebida na profissão } \\
\text { (KMO: } 0,919 ; \text { Teste de Bartlett: } \chi^{2}=948,49, p<0,001 \text {; Variância extraída: } 70,84 \% \text { ) }\end{array}$} \\
\hline $\begin{array}{l}\text { A profissão de administrador } \\
\text { tem uma boa reputação no } \\
\text { mercado }\end{array}$ & ,823 & 6,86 & 7,00 & 2,20 &,- 61 &,- 01 \\
\hline $\begin{array}{l}\text { Meu diploma de administrador } \\
\text { tem um bom valor em termos } \\
\text { profissionais }\end{array}$ & ,748 & 7,65 & 8,00 & 2,14 & $-1,10$ & 1,19 \\
\hline $\begin{array}{l}\text { Eu acredito que os } \\
\text { empregadores avaliam } \\
\text { positivamente a profissão de } \\
\text { administrador }\end{array}$ & 873 & 6,99 & 8,00 & 2,32 &,- 90 & ,40 \\
\hline $\begin{array}{l}\text { De forma geral, vejo o nível } \\
\text { de reputação de mercado da } \\
\text { profissão de administrador como }\end{array}$ & ,884 & 6,93 & 7,00 & 1,94 & $-1,24$ & 1,74 \\
\hline $\begin{array}{l}\text { Comparativamente com outras } \\
\text { profissões, a profissão de } \\
\text { administrador tem um bom } \\
\text { prestígio externo }\end{array}$ & ,898 & 6,52 & 7,00 & 2,24 &,- 75 & 19 \\
\hline $\begin{array}{l}\text { Em termos profissionais, tenho } \\
\text { segurança de que serei feliz } \\
\text { como administrador }\end{array}$ & 711 & 7,43 & 8,00 & 2,14 & $-1,06$ & ,65 \\
\hline $\begin{array}{l}\text { O mercado tem muito respeito } \\
\text { por minha futura profissão }\end{array}$ & ,931 & 6,43 & 7,00 & 2,18 &,- 63 &,- 01 \\
\hline \multicolumn{7}{|c|}{$\begin{array}{l}\text { Identificação pessoal com a profissão } \\
\text { (KMO: 0,863; Teste de Bartlett: } x^{2}=814,74, p<0,001 \text {; Variância extraída: } 81,03 \% \text { ) }\end{array}$} \\
\hline $\begin{array}{l}\text { Tenho total segurança quanto } \\
\text { a minha identificação com a } \\
\text { profissão de administrador }\end{array}$ & ,892 & 7,58 & 8,00 & 2,22 & $-1,17$ & 1,10 \\
\hline $\begin{array}{l}\text { Eu tenho clareza de minha } \\
\text { identificação com a minha futura } \\
\text { profissão }\end{array}$ & ,866 & 8,01 & 8,00 & 2,05 & $-1,36$ & 1,80 \\
\hline $\begin{array}{l}\text { Tenho verdadeira identificação } \\
\text { com a profissão de administrador }\end{array}$ & 941 & 7,63 & 8,00 & 1,94 & $-1,16$ & 1,54 \\
\hline $\begin{array}{l}\text { Eu sou seguro da minha } \\
\text { identificação com o curso e com } \\
\text { a carreira de administrador }\end{array}$ & ,896 & 7,73 & 8,00 & 2,14 & $-1,23$ & 1,40 \\
\hline $\begin{array}{l}\text { Em geral, meu nível de } \\
\text { identificação com a profissão de } \\
\text { administrador é }\end{array}$ & 905 & 7,95 & 8,00 & 1,70 & $-1,11$ & 1,18 \\
\hline
\end{tabular}




\section{DADOS DOS AUTORES}

\section{STEPHANIE INGRID SOUZA BARBOZA^ stephanieisb@gmail.com} Doutoranda pela UFPB

Professora na UFB

Instituição de vinculação: Universidade Federal da Paraíba

João Pessoa/PB - Brasil

Áreas de interesse em pesquisa: Marketing; Métodos Quantitativos em Administração; Educação em Administração.

* Universidade Federal da Paraíba Cidade Universitária João Pessoa/PB 58051-900

\section{DIANA LÚCIA TEIXEIRA DE CARVALHO dinda_carvalho@hotmail.com} Mestre em Administração pela UFPB

Instituição de vinculação: Universidade Federal da Paraíba

João Pessoa/PB - Brasil

Áreas de interesse em pesquisa: Marketing; Métodos Quantitativos em Administração;

Educação em Administração.

\section{JOÃO BATISTA SOARES NETO jnetojp@gmail.com} Mestre em Administração pela UFRN

Instituição de vinculação: Universidade Federal da Paraíba João Pessoa/PB - Brasil

Áreas de interesse em pesquisa: Marketing; Métodos quantitativos em Administração;

Educação em Administração.

\section{FRANCISCO JOSÉ DA COSTA franzecosta@gmail.com}

Doutor em Administração pela FGV/EAESP

Instituição de vinculação: Universidade Federal da Paraíba

João Pessoa/PB - Brasil

Áreas de interesse em pesquisa: Marketing; Métodos quantitativos em Administração;

Educação em Administração. 